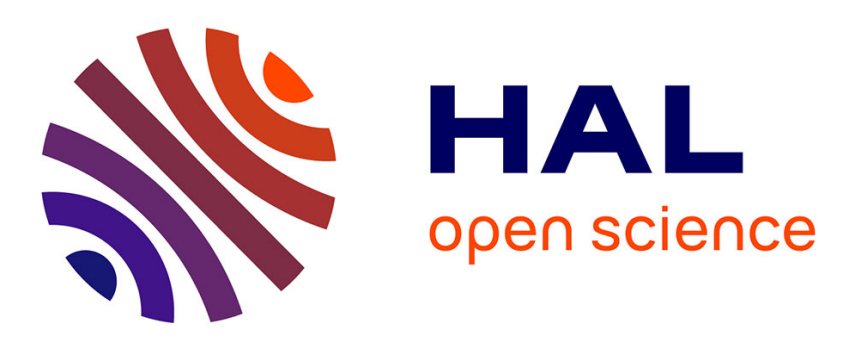

\title{
3D Curves Tracking and its Application to Cortical Sulci Detection
}

\author{
Christophe Renault, Michel Desvignes, Marinette Revenu
}

\section{To cite this version:}

Christophe Renault, Michel Desvignes, Marinette Revenu. 3D Curves Tracking and its Application to Cortical Sulci Detection. IEEE International Conference on Image Processing, Sep 2000, Vancouver, Canada. pp.491-494. hal-00821982

\section{HAL Id: hal-00821982 https://hal.science/hal-00821982}

Submitted on 13 May 2013

HAL is a multi-disciplinary open access archive for the deposit and dissemination of scientific research documents, whether they are published or not. The documents may come from teaching and research institutions in France or abroad, or from public or private research centers.
L'archive ouverte pluridisciplinaire HAL, est destinée au dépôt et à la diffusion de documents scientifiques de niveau recherche, publiés ou non, émanant des établissements d'enseignement et de recherche français ou étrangers, des laboratoires publics ou privés. 


\title{
3D CURVES TRACKING AND ITS APPLICATION TO CORTICAL SULCI DETECTION
}

\author{
Christophe Renault, Michel Desvignes, Marinette Revenu \\ GREYC - ISMRA, 6, Boulevard Maréchal Juin, \\ 14050 CAEN Cedex, FRANCE \\ e-mail : christophe.renault@greyc.ismra.fr
}

\begin{abstract}
Automatic labelling and identification of cerebral structure, such as cortical sulci, are useful in neurology, surgery planning, etc... In this paper, a method to detect the valley of the cortical sulci is presented. The aim of the method is to achieve the sulci medial surface. The method applied on MRI data, is based on geometrical features (curvature) which doesn' t require the accurate segmentation of the cerebral cortex. We use a sub-voxel precision tracking. The minimum curvature vector in each point allows successive displacement along the valley of sulci. Partial derivatives provide the differential characteristics.
\end{abstract}

\section{INTRODUCTION}

Human cortical sulci identification on 3D image is used in neurology, surgical planning, human brain mapping... Cortical sulci are landmarks and represent gross morphology to localise anatomical structures and functional areas with respect to these landmarks. Sulci are deep cortical folds and previous works are the automatic labelling of the superficial trace of the sulci on the surface of the brain [3]. This paper presents a method to detect the deep cortical fold without an accurate segmentation of brain tissue (white matter, grey matter, cerebro-spinal fluid), using curvature features. In fig. 1, an interactive drawing of the medial surface of sulci is superimposed with the MRI slice. Unlike works which start from the outer edge of sulcus to go toward the valley [5], we want to propagate the valley toward the outer edge, in the opposite path [6]. Then, the sulcus valley detection is an important stage of the process. This detection is obtained by 3 dimensions curve tracking.

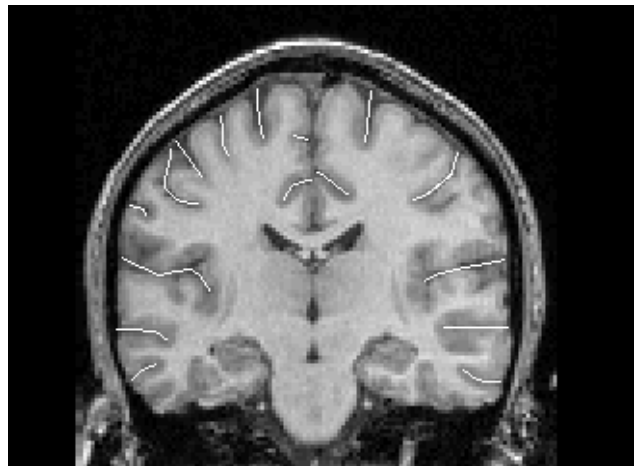

Figure 1: Medial surface of sulci superimposed with the MRI slice.

\section{SULCI AND CURVATURE}

Cortical sulci and gyri define gross anatomical landmarks on the surface of the cerebral cortex. Sulci represent the cortical folds. The parts between these convolutions are the gyri. Physical limits between sulci and gyri are not precisely defined, but respectively square with concave and convex parts. Curvature is then used to separate sulci and gyri. In 3D images, at each point A of an iso-surface $I$, there are two principal directions $\vec{t}_{1}$ and $\vec{t}_{2}$ in tangent plane of $I$, which correspond to the maximal and minimal curvature $k_{1}$ and $k_{2}$ (fig. 2) [4].

$K$ and $S$ are the gaussian and average curvature:

$$
\begin{gathered}
K=k_{1} k_{2} \\
S=\frac{k_{1}+k_{2}}{2}
\end{gathered}
$$

From differential characteristics of the image we compute $K$ and $\mathrm{S}$ as following: 


$$
\begin{gathered}
K=\frac{1}{h^{2}}\left(\begin{array}{c}
I_{x}^{2}\left(I_{y y} I_{z z}-I_{y z}^{2}\right)+2 I_{y} I_{z}\left(I_{x z} I_{x y}-I_{x x} I_{y z}\right)+ \\
I_{y}^{2}\left(I_{z z} I_{y y}-I_{x z}^{2}\right)+2 I_{z} I_{x}\left(I_{x y} I_{y z}-I_{y y} I_{x z}\right)+2 I_{x} I_{y}\left(I_{y z} I_{x z}-I_{z z} I_{x y}\right)
\end{array}\right) \\
S=\frac{1}{2 h^{3 / 2}}\left(\begin{array}{l}
I_{x}^{2}\left(I_{y y}+I_{z z}\right)-2 I_{y} I_{z} I_{y z}+ \\
I_{y}^{2}\left(I_{z z}+I_{x x}\right)-2 I_{z} I_{x} I_{x z}+ \\
I_{z}^{2}\left(I_{x x}+I_{y y}\right)-2 I_{x} I_{y} I_{x y}
\end{array}\right)
\end{gathered}
$$

with $h=I_{x}^{2}+I_{y}^{2}+I_{z}^{2}$

and $I_{a^{n} b^{m}}=\frac{\partial^{n+m}(G(a, b) \otimes I)}{\partial a^{n} \partial b^{m}}$

where $G(a, b)=\frac{1}{\sigma \sqrt{2 \pi}} e^{-\frac{a^{2}+b^{2}}{2 \sigma^{2}}}$

and $\otimes$ the convolution operator.

$k_{1}$ and $k_{2}$ are then obtained by:

$$
k_{1,2}=S \pm \sqrt{S^{2}-K}
$$

and directions $\vec{t}_{1}$ and $\vec{t}_{2}$ by:

$$
\overrightarrow{t_{1,2}}=\vec{\alpha} \pm \sqrt{S^{2}-K} \vec{\beta}
$$

with $\vec{\beta}=\left(I_{z}-I_{y}, I_{x}-I_{z}, I_{y}-I_{x}\right)$ and

$$
\vec{\alpha} \cdot \vec{x}=-\frac{1}{2 h^{3 / 2}}\left(\begin{array}{l}
-2 I_{z}^{3} I_{x y}+I_{y}^{3} I_{z z}+2 I_{y}^{3} I_{x z}-2 I_{y}^{2} I_{z} I_{x y} \\
+2 I_{z}^{2} I_{x} I_{y z}+2 I_{z}^{2} I_{y} I_{x z}-2 I_{y}^{2} I_{x} I_{y z} \\
-2 I_{x} I_{y} I_{z} I_{z z}+2 I_{x} I_{y} I_{z} I_{y y}+I_{y}^{2} I_{z} I_{x x} \\
-2 I_{z}^{2} I_{x} I_{x z}+I_{x}^{2} I_{z} I_{z z}-I_{x}^{2} I_{z} I_{y y}+2 I_{z}^{2} I_{y} I_{y z} \\
-I_{y}^{2} I_{z} I_{z z}+I_{z}^{3} I_{x x}-I_{z}^{3} I_{y y}-2 I_{y}^{2} I_{x} I_{x z} \\
+2 I_{x}^{2} I_{y} I_{y z}-I_{y}^{3} I_{x x}+2 I_{z}^{2} I_{x} I_{x y}-I_{z}^{2} I_{y} I_{x x} \\
-2 I_{y}^{2} I_{z} I_{y z}+I_{z}^{2} I_{y} I_{y y}-2 I_{x}^{2} I_{z} I_{y z} \\
+2 I_{y}^{2} I_{x} I_{x y}+I_{x}^{2} I_{y} I_{z z}-I_{x}^{2} I_{y} I_{y y}
\end{array}\right)
$$

The $y$ an $z$ components of the vector $\vec{\alpha}$ are obtained by circular permutations of $x, y$ and $z$.

In case of sulci, $\vec{t}_{2}$ is along the valley. $\vec{t}_{2}$ will be used to follow the roof of sulci.

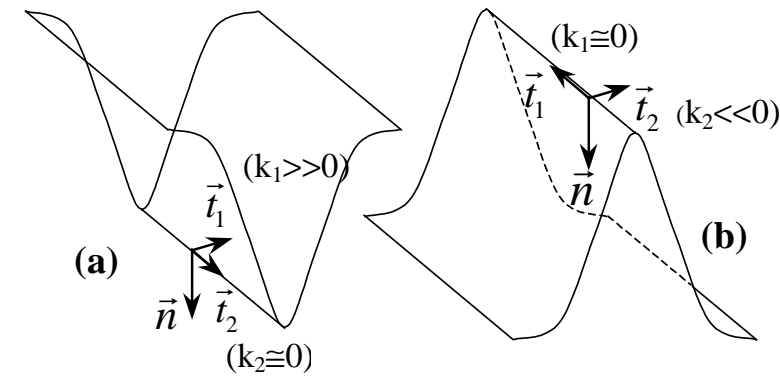

Figure 2: (a) Principal curvature on sulci, (b) Principal curvature on gyri.

\section{3D CURVE TRACKING}

Sulci have to be represented as a parametric 3D curve, i.e. an ordered set of connected voxels $(x(t), y(t), z(t))$. The basic idea of the tracking process is to use the $\vec{t}_{2}$ vector, which give the direction of the next voxel for each point of the surface.

To achieve an iterative crest line tracking, we need three notions:

- Starting points, origin of 3D curve.

- A function which determines the next point of the curve, in local neighbourhood.

- A condition to stop the iterative process.

\subsection{Discrete case}

In the 3D discrete grid of the image we have noticed two main problems.

- In the direction of $\vec{t}_{2}$, a voxel $V$ is chosen. Now, there is only a finite number (26) of directions defined by the local neighbourhood.

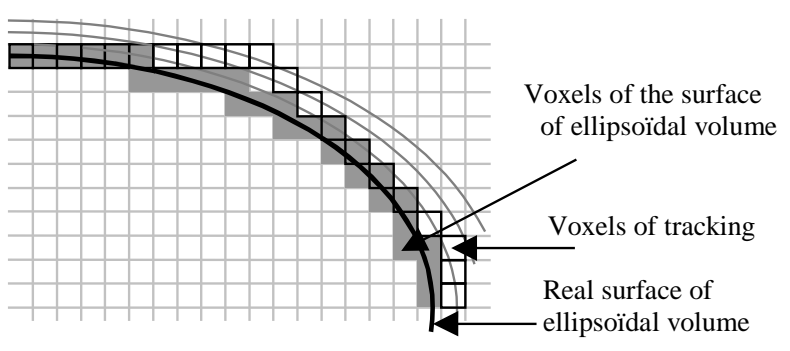

Figure 3: Discrete tracking

The tracking only moves in horizontal, vertical and diagonal directions (Fig.4 ) because of this restrictive choice of next voxels.

- Local curvature values are different from real values.

These two points lead the tracking process to fail to detect and to extract the sulci. 


\subsection{Subvoxel tracking}

Since discrete derivative value are not accurate enough to ensure a precise detection, we used a sub-voxel tracking. Voxel notion is replaced by point notion, since the tracking goes step by step with a step smaller than voxel. The point positions are not in the grid of volume, so the differential characteristics are computed by trilinear or better interpolation.

\section{$\underline{\text { Starting points }}$}

First, MLvv operator [1][2], similar to average curvature, is computed. Negative values of MLvv are kept by thresholding. The image is labelled and each connected component is a volume representing one sulcus. The starting points are the voxels with the highest curvature in each connected component.

\section{Following points}

Several functions to determine the next point have been examined. 3 of them are now presented.

\section{Case 1:}

From a point $P$, the next point $N$ is the point in direction of the $\vec{t}_{2}$ vector, at the distance step. This method is adequate in case of simple structures. Fig. 4 presents the results on an ellipsoidal structure. The first point of the tracking has been manually chosen. The result is the expected crest line.

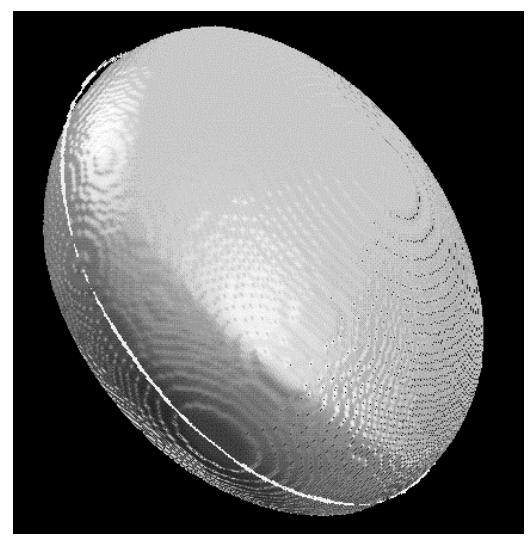

Figure 4: In white, the line corresponding to tracking on ellipsoïdal structure.

Case 2:

To deal with approximate value of curvature, the direction is a linear combination between $\vec{t}_{2}$ and the direction of maximal curvature. A new direction $\vec{r}$ toward the maximal average curvature is added to $\vec{t}_{2}$.

$$
\begin{array}{ll} 
& \vec{d}=\alpha \overrightarrow{t_{2}}+(1-\alpha) \vec{r} \\
\text { where } \quad \vec{r} & =\sum_{i=1}^{8} \frac{\overrightarrow{P M_{i}} C_{i}}{\left\|P M_{i}\right\|}
\end{array}
$$

$M_{i}$ are the 8 neighbour voxels of $P, C_{i}$ their respective curvature. This method is adequate with large object without very high curvature values and good SNR. With large curvature, $\vec{t}_{2}$ and $\overrightarrow{\boldsymbol{r}}$ are sometimes opposite, and $\vec{d}$ null. So the tracking process stops before the real termination

Case 3:

To avoid the previous problem, $\vec{r}$ belong to the normal plan of $\vec{t}_{2}$ (Fig.5). The effect of $\vec{t}_{2}$ must be predominant

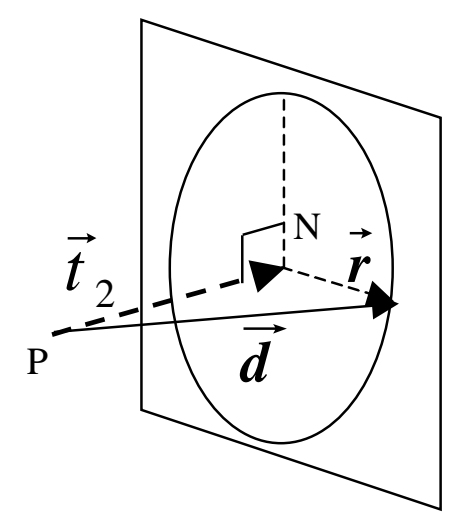

Figure 5: Direction $\vec{r}$ in normal plan of $\overrightarrow{\boldsymbol{t}}_{2}$

\section{Stopping conditions}

This process is stopped when:

- The next voxel is in a gyri area (positive average curvature value).

- Umbilical area is reached: the principal curvatures $K_{1}$ and $K_{2}$ are equal, $\vec{t}_{1}$ and $\vec{t}_{2}$ are not defined in differential geometry.

- The curvatures are not defined: in area of null gradient values.

- A junction with an other sulcus.

\section{RESULTS}

MRI of healthy volunteers were acquired on a 1.5 Tesla GE Signa scanner using a SPGR sequence $(124 * 256 * 256$ voxels, $1.3 \mathrm{~mm} 3)$. 


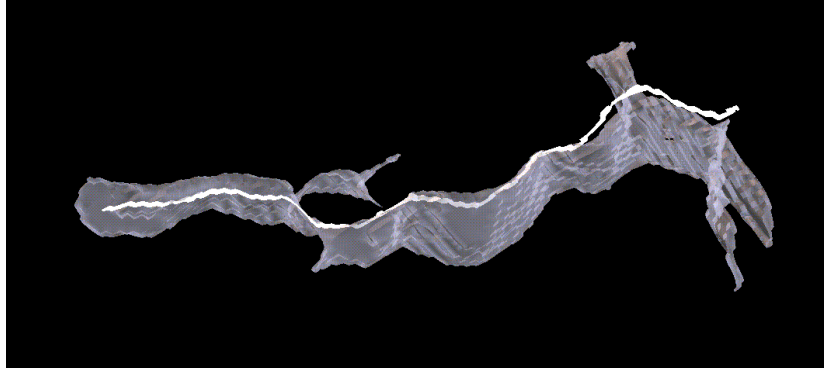

Figure 6: Result on a sulcus

Fig. 6 shows the result on a single sulcus. The white line is the result of the sub-voxel tracking (case 3). The grey part is a sulcus segmentation done by threshold of maximal curvature. On this Fig. 6. The tracking doesn't seem to be, in each point, on the roof of sulcus, because of viewpoints effect and non accurate segmentation of sulcus. However, replaced on the raw MRI slices, the tracking is visually located on the roof of sulcus.

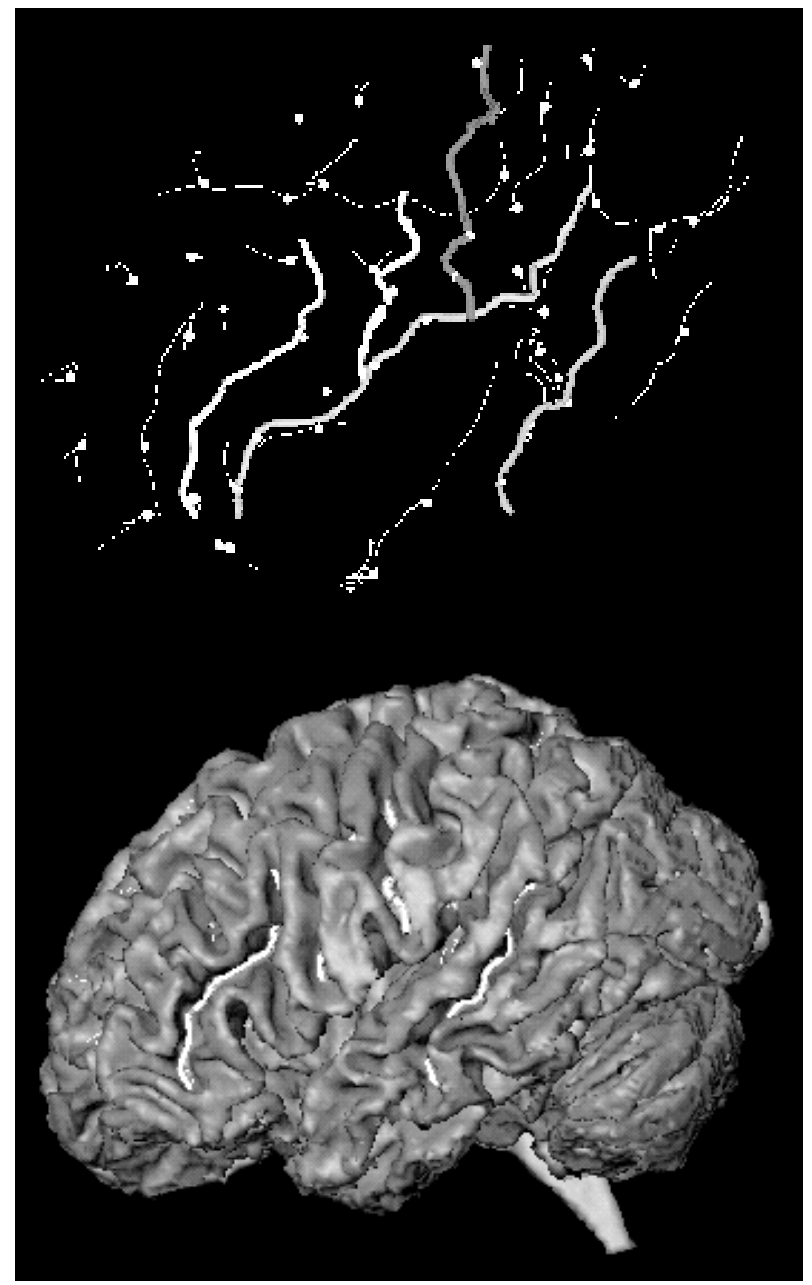

Figure 7: Tracking results on one hemisphere. Without (top) and with (bottom) superimposed segmentation of the cortex.
All the lines corresponding to the sulci valley on a single hemisphere of a brain are visualized on Fig. 7. The bottom image shows the results of multiple sulci superimposed with a segmentation of the brain.

\section{CONCLUSION}

We have presented a method to extract roof of sulci with a sub-voxel precision. This method uses MRI brain data without accurate segmentation of the cerebral cortex. Roof of sulci are represented as 3D parametric curve, these curves are easy to statistically handle (i.e. average curve, standard deviation) and are used to define an atlas of cerebral sulci.

\section{REFERENCES}

[1] L. M. J. Florack, B. M. ter Haar Romeny, J. J. Koenderink, M. A. Viergever. "Scale and differential structure of images", Image and Vision Computing, Vol 10, July/August 1992, p 376388 .

[2] G. Le Goualher, C. Barillot, Y. Bizais, J-M Scarabin. "3D segmentation of cortical sulci using active models". SPIE Proceedings of Medical Imaging, Image Processing, 1996, volume 2710, p 254-263.

[3] N. Royackkers. "Modélisation et reconnaissance des Sillons du cortex cérébral humain". Ph.D Thesis, University of Caen, France, 1997.

[4] J.P. Thirion, A. Gourdon, "The Marching Lines Algorithm: new results and proofs". INRIA, Research report $n^{\circ} 1881$, April 1993.

[5] G. Le Goualher, C. Barillot, Y. Bizais. "Modeling Cortical Sulci with Active Ribbons". Int. J. of Pattern Recognition and Artificial Intelligence, 1997, Vol 11(8), pp. 1295-1315.

[6] M. Vaillant, C. Davatzikos, R. N. Bryan, "Finding 3D Parametric Representations of the Deep Cortical Folds". Proc. of the IEEE Workshop on Mathematical Methods, Biomedical Image Analysis, June 1996, pp 151-159. 